\title{
Extrusão do anel intra-estromal corneano e vascularização do túnel
}

\section{Extrusion and vascularization of the intrastromal corneal ring tunnel}

Larissa Casteluber', Jaquison Furtado da Silva' ${ }^{2}$, Hilton Arcoverde Gonçalves de Medeiros ${ }^{3}$

\section{Resumo}

O objetivo deste trabalho é relatar e discutir os aspectos de um caso clínico em que foi observada a formação de neovascularização no túnel do anel intra-estromal corneano. Trata-se de paciente com ectasia corneana 4 anos após LASIK, comprovada pela paquimetria e topografia, e submetido ao implante de anel intra-estromal corneano. No terceiro ano de acompanhamento após implante do anel intraestromal, com o paciente em uso de lente de contato gelatinosa, verificou-se extrusão de um segmento e neovascularização no túnel. Removeu-se o segmento afetado, realizou-se fotocoagulação vascular, observando-se regressão completa do quadro neovascular.

Descritores: Estroma corenano/physiopathologia; Estroma corneano/cirurgia; Neovascularização da córnea; Próteses e implantes Córnea/patologia; Fotocoagulação; Relato de casos [Tipo de publicação]

\footnotetext{
1 Médico-oftalmologista da Clínica de Olhos Dr. João Eugenio - Brasília (DF), Brasil;

${ }^{2}$ Médico-oftalmologista da Clínica de Olhos Dr. João Eugenio - Brasília (DF), Brasil;

Doutor em Oftalmologia pela UFG, Médico-oftalmologista da Clínica de Olhos Dr. João Eugenio - Brasília (DF), Brasil.

Trabalho realizado na Clínica de Olhos Dr. João Eugenio, Brasília (DF), Brasil.
}

Recebido para publicação em: 17/07/2007 - Aceito para publicação em 6/12/2007 


\section{INTRODUÇÃO}

$\mathbf{O}$ Anel intra-estromal é uma órtese que tem como objetivo regularizar a curvatura corneana e, assim sendo, diminuir os erros refracionais decorrentes da irregularidade das córneas ectásicas, podendo melhorar o efeito da correção ótica com óculos ou lentes de contato ${ }^{(1-2)}$.

O anel é fabricado a partir do PMMA (polimetilmetacrilato), material comprovadamente inerte e biocompatível que é utilizado há décadas na fabricação de implantes intra-oculares ${ }^{(3)}$. É indicado principalmente aos portadores de ceratocone com baixa acuidade visual, intolerância às lentes de contato, e indicação de transplante de córnea ${ }^{(1-4)}$. Este tratamento cirúrgico tem sido utilizado também nos casos de ectasia corneana secundária a cirurgia fotorrefrativa com excimer laser ${ }^{(5)}$.

Estudos demonstram que este é um método seguro e efetivo, com bons resultados visuais e topográficos, principalmente nos casos não avançados de ectasia (até $60 \mathrm{D})^{(5-7)}$.

A incidência de complicações varia entre os estudos. Entre as principais complicações descritas estão: deslocamento do segmento do anel intraestromal; extrusão; posicionamento assimétrico; ceratite infecciosa; hidropsia aguda; depósitos no interior do túnel estromal; além dos sintomas visuais como halos e glare ${ }^{(8)}$.

Neste artigo descrevemos a ocorrência da vascularização de parte do túnel estromal associada à extrusão do segmento, e conduta terapêutica empregada.

\section{Relato do caso}

Em agosto de 2004, foi atendido VJS, 24 anos, masculino, branco, com instabilidade visual mesmo em uso de lentes corretivas. Relata ter feito cirurgia refrativa há 4 anos (LASIK). Ao exame apresentava refração em OD: - 16.25 Esf - 3.00 Cil $56^{\circ}=20 / 80$ e em OE: -3.50 Esf $-4.50 \mathrm{Cil} 112^{\circ}=20 / 40$. Paquimetria: OD: $0.343 \mathrm{~mm} \mathrm{OE:}$ $0.391 \mathrm{~mm}$. A topografia revela ectasia corneana bilateral com quadro avançado em OD (Figura 1).

Foi indicado para esse paciente o implante de anel intra-estromal em ambos os olhos. No pós-operatório imediato, os anéis encontravam-se muito bem posicionados e o paciente relatava uma melhora acentuada na acuidade visual. Sua refração após o implante do anel era: OD: -5.00 Esf $-2.00 \mathrm{Cil} 75^{\circ}=20 / 30$, e OE: 1.25 Esf -2.00 Cil $180^{\circ}=20 / 30$.

Em junho de 2005, retorna com quadro estável utilizando lentes de contato gelatinosas tóricas da marca Focus Toric ${ }^{\circledR}$ (Vifilcon A, CIBA Vision, USA), com acuidade visual permanecendo em 20/30 em ambos os olhos. As lentes de contato possuíam a seguinte graduação: $\mathrm{OD}=-4.50 \mathrm{Esf}-1.75 \mathrm{Cil} 90^{\circ}$ e $\mathrm{OE}=-1.25 \mathrm{Esf}-1.75$ Cil $180^{\circ}$ e o paciente fazia uso diário das mesmas, removendo-as à noite.

Paciente retorna a esse serviço em maio de 2007, para uma revisão periódica, estando assintomático. No

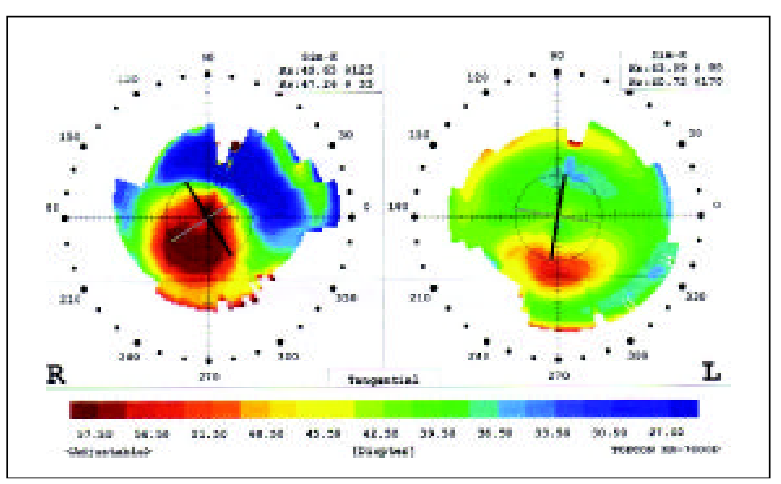

Figura 1: Topografia corneana prévia a colocação dos anéis estromais

exame clínico verificou-se uma intensa neovascularização ao nível do túnel do anel intra-estromal, em OD com extrusão da extremidade medial do anel (Figuras 2 e 3). No OE não foi observada nenhuma alteração. A conduta nesse caso foi a retirada imediata do segmento do anel no OD, com realização de fotocoagulação com laser de argônio verde no vaso nutridor da neovascularização corneana.

Sob biomicroscopia, e utilizando-se de magnificação de 16x e retroiluminação, foi identificado o vaso aferente da neovascularização. Foi realizada a fotocoagulação, primeiro do vaso aferente, seguido pelo vaso eferente, utilizando-se os parâmetros e técnica definidos por Sharma et al. ${ }^{(9)}$.

O paciente evoluiu com remissão completa da neovascularização em OD, porém com prejuízo da refração a qual estava em $-18.50-3.25174^{\circ}=20 / 80$. Encontra-se hoje aguardando para tentar reposicionamento de anel com 6 meses.

\section{Discussão}

O erro refracional causado por ectasia da córnea é inicialmente compensado por óculos ou lente de contato. Quando a ectasia progride a um ponto em que as lentes não promovem melhora satisfatória da visão, a intervenção cirúrgica pode ser considerada ${ }^{(8,10)}$.

A ceratoplastia penetrante é a mais comum intervenção cirúrgica para a resolução da ectasia corneana, porém, está associada com complicações incluindo rejeição do botão doador, indução ao astigmatismo, complicações intra-oculares como o glaucoma, formação de catarata, descolamento de retina, edema macular cistóideo, endoftalmites e hemorragias expulsivas. Para contornar essas complicações, novos métodos como a ceratoplastia lamelar e o implante de segmentos intraestromais corneanos foram desenvolvidos ${ }^{(7,11)}$.

O tratamento do ceratocone e da ectasia pós-cirurgia refrativa com o uso do anel intra-estromal tem se revelado um bom método, pois promove o aplanamento central da córnea, possibilitando ao paciente o uso de óculos, lentes de contato gelatinosas tóricas, ou lentes 


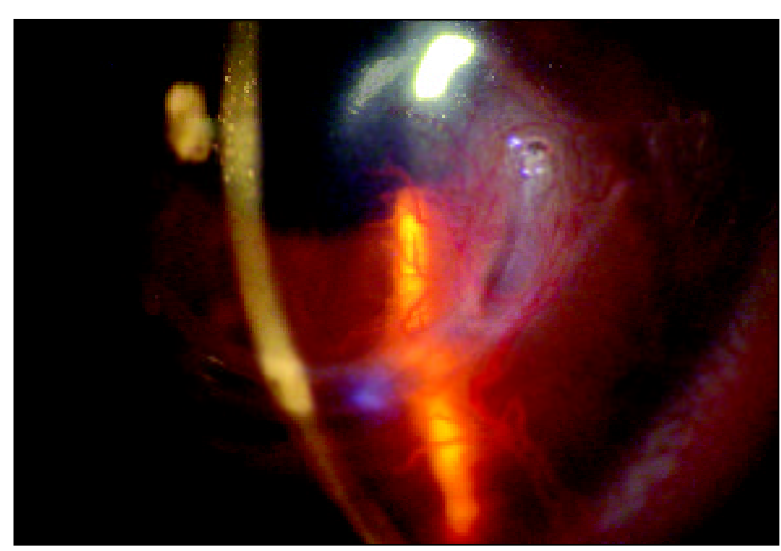

Figura 2: Segmento de anel com extrusão da extremidade e neovascularização do túnel

gás-permeáveis. Assim sendo, permite postergar ou evitar o transplante de córnea ${ }^{(4,6,8)}$.

Os anéis estromais são usados na correção de baixa miopia, no ceratocone e na ectasia pós-cirurgia refrativa com excimer laser.

Algumas complicações pós-implante foram relatadas, sendo de caráter benigno e reversíveis após a retirada do anel. As mais comuns foram a descentralização do segmento $(2.7 \%)$, posicionamento assimétrico $(5 \%)$, migração (5\%), extrusão (13.8\%), conjuntivite $(2.7 \%)$, ceratite bacteriana $(2.7 \%)$ e hidropsia $(2.7 \%)^{(1)}$.

A vascularização profunda do túnel do anel é uma das complicações possíveis. Normalmente não associada ao sítio da incisão, necessita sempre da remoção do segmento afetado. Nestes casos a regressão dos neovasos é a regra ${ }^{(12)}$.

A fotocoagulação em neovasos corneanos é uma técnica segura e eficaz ${ }^{(9)}$, capaz de diminuir a área da vascularização corneana em olhos com opacidades corneanas vascularizadas.

Apesar de o implante do anel intra-estromal ser um procedimento seguro, eficaz, e apresentar bons resultados, complicações podem existir e o paciente deve ser avaliado periodicamente, sendo que qualquer alteração deve ser tratada imediatamente, avaliando-se a necessidade da retirada do segmento ${ }^{(8)}$.

\section{Abstract}

The purpose of this paper is to describe the clinical aspects of one case with deep corneal vascularization after corneal ring implantation to treat corneal ectasia due to LASIK 4 years before. The corneal ectasia diagnostic was performed by corneal pachimetry and topography. Intrastromal corneal ring segment was implanted. On the third year of follow-up, extrusion of one segment was noted and deep corneal neovascularization was found. The segment was removed, laser photocoagulation was applied and complete vascular regression was observed.

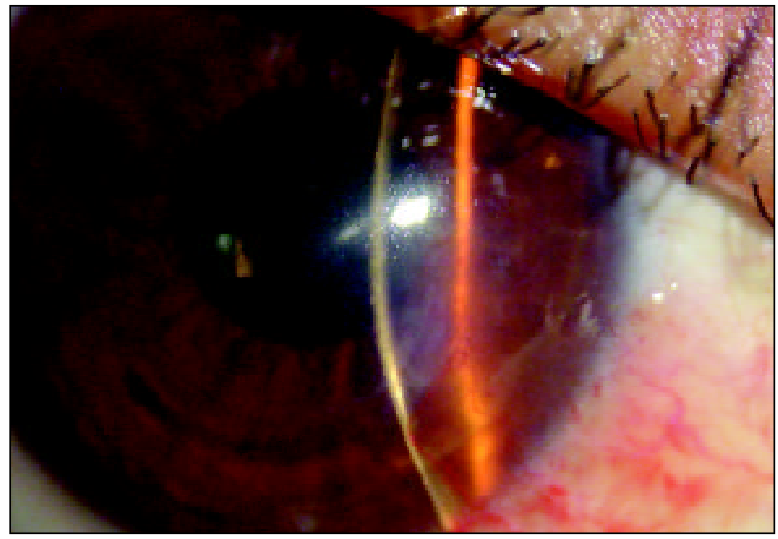

Figura 3: Após retirada do anel, regressão dos neovasos

Keywords: Corneal stroma/physiopathology; Corneal stroma/surgery; Corneal neovascularization; Prostheses and Implants; Cornea/pathology; Light coagulation; Case reports [Publication type]

\section{RefERÊNCIAS}

1. Miranda D, Sartori M, Francesconi C, Allemann N, Ferrara P, Campos M. Ferrara intrastromal corneal ring segments for severe keratoconus. J Refract Surg. 2003; 19(6):645-53.

2. Tan DT, Por YM. Current treatment options for corneal ectasia. Curr Opin Ophthalmol. 2007; 18(4):284-9.

3. D'Hermies F, Hartmann C, von Ey F, Holzkämper C, Renard G, Pouliquen Y. Biocompatibility of a refractive intracorneal PMMA ring. Fortschr Ophthalmol. 1991; 88(6):790-3.

4. Siganos D, Ferrara P, Chatzinikolas K, Bessis N, Papastergiou G. Ferrara intrastromal corneal rings for the correction of keratoconus. J Cataract Refract Surg. 2002; 28(11):1947-51.

5. Binder PS. Ectasia after laser in situ keratomileusis. J Cataract Refract Surg. 2003; 29(12):2419-29. Review.

6. Hofling-Lima AL, Branco BC, Romano AC, Campos MQ, Moreira H, Miranda D, et al. Corneal infections after implantation of intracorneal ring segments. Cornea. 2004; 23(6):547-9. Erratum in: Cornea. 2004; 23(7): table of contents. Casanova, Fabio [corrected to Casanova, Fabio H].

7. Tan BU, Purcell TL, Torres LF, Schanzlin DJ. New surgical approaches to the management of keratoconus and postLASIK ectasia. Trans Am Ophthalmol Soc. 2006; 104:21220.Kwitko S,

8. Severo NS. Ferrara intracorneal ring segments for keratoconus. J Cataract Refract Surg. 2004; 30(4):812-20.

9. Sharma A, Samal A, Narang S, Gutpa A, Ram J, Gupta A. Frequency doubled Nd:YAG $(532 \mathrm{~nm})$ laser photocoagulation in corneal vascularisation: efficacy and time sequenced changes. Indian J Ophthalmol. 2001; 49(4):235-40.

10. Kaiura TL, Seedor JA, Koplin RS, Rhee MK, Ritterband DC, Lipton EJ. Subepithelial intracorneal hemorrhage in a soft contact lens user. Eye Contact Lens. 2004; 30(3):120-1.

11. Shabayek MH, Alió JL. Intrastromal corneal ring segment implantation by femtosecond laser for keratoconus correction. Ophthalmology. 2007; 114(9):1643-52.

12. Al-Torbak A, Al-Amri A, Wagoner MD. Deep corneal neovascularization after implantation with intrastromal corneal ring segments. Am J Ophthalmol. 2005; 140(5):926-7. 